DOI: https://doi.org/10.46630/phm.12.2020.22

Sanja B. Bošković-Danojlić ${ }^{1}$

Article de recherche

Université de Poitiers

Faculté des lettres et des langues

УДК 378.18(=163.41)(44)"1914/1918"

Département des langues slaves et orientales

316.722(497.11:44)"1914/1918"

Groupe de recherche : MIMMOC EA3812

Reçu : le 29/2/2020

\title{
LES ÉTUDIANTS SERBES À L'UNIVERSITÉ DE POITIERS PENDANT LA PREMIÈRE GUERRE MONDIALE
}

\begin{abstract}
Concernant la France, la terre d'accueil pour des émigrants serbes et, ensuite yougoslave, on peut dire que leur nombre variait selon les différentes époques. Le moment de l'intensification de la fluctuation migratoire serbe, comprenant surtout la jeune population - les élèves et les étudiants - en direction de la France, s'effectua lors de la Première Guerre mondiale. À la suite de la retraite de l'armée et l'exil du peuple serbes traversant l'Albanie durant l'hiver 1915/1916, le flux migratoire des élèves serbes va particulièrement accroître en 1916. La ville de Poitiers, ses collèges et lycées ainsi que l'Université de Poitiers vont offrir l'hospitalité aux jeunes réfugiés serbes. Les archives de l'Association générale des étudiants de Poitiers, fondée le 7 juin 1889, montre non seulement la fluctuation des écoliers et des étudiants serbes pendant la Grande Guerre, mais aussi témoignent d'une période historique bien précise dans les relations politiques et culturelles franco-serbes.
\end{abstract}

Mots clés : Grande Guerre, réfigiés serbes, étudiants serbes, Poitiers

\section{Introduction}

Au cours du siècle dernier, l'espace des Balkans s'avère comme un espace touché par l'émigration. Ce sont les raisons économiques qui poussent, dès la fin du XIX $\mathrm{X}^{\mathrm{e}}$ siècle, les paysans et les éleveurs paupérisés à « chercher du travail dans les centres urbains ou à partir à l'étranger » (MOROKVAŠIĆMÜLLER 1998 : 154). La principale destination des migrants de cette région, après les bassins miniers en France, en Belgique ou en Allemagne, fut l'Amérique du Nord. Cependant, au cours des années vingt du $\mathrm{XX}^{\mathrm{e}}$ siècle, on constate que la crise économique et la récession frappant l'économie des Etats-Unis réoriente le flux migratoire vers les pays européens.

Concernant la France, la terre d'accueil pour des émigrants serbes et, ensuite yougoslave, on peut dire que leur nombre variait selon les différentes

1 sanja.boskovic@univ-poitiers.fr 
époques allant « de 4032 en 1921 » et passant à « 31873 en 1931 ; il a chuté à 20000 avant la Seconde Guerre mondiale et s'est maintenu à ce niveau jusqu'aux années soixante, alors qu'il monte à 47544 en 1968 et à 70280 en 1975 (MOROKVAŠIĆ-MÜLLER 1998 : 154). Selon Mirjana MorokvašićMüller, le nombre de migrants d'origine de l'espace ex-yougoslave en France « depuis vingt ans [...] s'est stabilisé et représente 85000 personnes environ » (MOROKVAŠIĆ-MÜLLER 1998 : 154).

Cependant parmi les dates marquant le renouveau dans les contacts franco-serbes, il faudrait mentionner l'année 1839 où le premier consul français arriva à Belgrade (VITANOVIĆ 2019). C'est aussi le moment lorsque « les premiers boursiers d'État, Filip Hristić, Dimitrije Crnobarac, Jovan Marinović et Konstantin Nikolajević, partirent à Paris pour y poursuivre des études de droit » (VITANOVIĆ 2019). En s'appuyant sur le modèle culturel français, la Serbie souhaite en effet afficher son attachement particulier envers les valeurs européennes qu'elle aspire intégrer. Ainsi le gouvernement serbe de l'époque manifesta son intérêt particulier de former sa future intelligentsia ${ }^{2}$ dans les meilleures universités françaises (VITANOVIĆ 2019) afin qu'elle puisse prendre un rôle considérable « dans l'organisation de l'État et dans la vie politique serbes » (VITANOVIĆ 2019).

L'année 1899 fut importante pour la création de la première chaire de langue et littérature françaises à Belgrade ce qui a non seulement contribué « grandement à l'accroissement de l'influence française sur la culture serbe dans de nombreux autres domaines »(VITANOVIĆ 2019) mais aussi a renforcé le flux migratoire des étudiants serbes vers la France.

\section{Grande Guerre et l'arrivée des élèves et des étudiants serbes en France}

Le moment de l'intensification de la fluctuation migratoire serbe, comprenant surtout la jeune population - les élèves et les étudiants - en direction de la France, s'effectua lors de la Première Guerre mondiale ${ }^{3}$. Selon

${ }^{2}$ «F. Hristić (1819-1905) qui fut à plusieurs reprises ministre, conseiller d'État, émissaire à Constantinople, Vienne, Londres, termina sa carrière en tant que premier gouverneur de la Banque nationale. D. Crnobarac (1818-1872) fut de même conseiller d'Etat, ministre de la justice et ministre de l'Enseignement, sans jamais toutefois entrer dans la diplomatie. J. Marinović (1821-1893) joua un rôle très important, notamment sous le règne du prince Mihailo et, plus largement, sous les Obrenović en tant que président du Conseil, ministre, chef du gouvernement, et représentant de la Serbie à Paris durant de nombreuses années. K. Nikolajević (1821-1877), qui épousa la fille du prince régnant Aleksandar, mit un terme relativement précoce à sa carrière diplomatique et ministérielle pour laisser un nom en tant qu'historien aux vastes connaissances » (VITANOVIĆ 2019).

${ }^{3}$ La France fut le premier des pays alliés à assister le Serbie financièrement, puis moralement et 
Miodrag Ibrovac, « dès le début même de la Grande Guerre, la France avait eu l'intention d'accueillir un certain nombre de nos élèves dans ses écoles » (IBROVAC 2014). Cependant, le transport organisé des jeunes serbes « commence à partir du mois de décembre 1915, transport du pays de la mort vers la terre du salut» (IBROVAC 2014).

Suite à la retraite de l'armée et l'exil du peuple serbes traversant l'Albanie durant l'hiver 1915/1916, le flux migratoire des élèves serbes va particulièrement accroître en 1916. D'après Slobodan Vitanović, la grande majorité de ces jeunes sera envoyée «en France pour y poursuivre leur scolarité »(VITANOVIĆ 2019). Vitanović distingue notamment l'absolu nécessité « pour l'État et la nation de disposer dans l'immédiat après-guerre d'une intelligentsia qui aurait pour devoir de remplacer les scientifiques et les intellectuels morts au combat »(VITANOVIĆ 2019). Selon lui, c'est la raison principale qui va guider « le gouvernement serbe, avec l'appui du gouvernement français, [...] d'évacuer les écoliers des zones de combats» (VITANOVIĆ 2019).

Selon les statistiques, le nombre de refugiés serbes se trouvant entre 1914-1918 sur le sol français varie selon les différentes sources. « Selon les sources serbes le nombre des Serbes en France aurait été autour de 30000 ; tandis qu'une des plus complètes Histoire de la population française indique que 17000 Serbes et 3000 Monténégrins séjournaient sur le sol français le 1er janvier 1918 »(TRGOVČEVIĆ 2010 : 364).

Parmi ces réfugiés, il y avait un nombre important des écoliers et des étudiants qui, suite à la décision du Ministère de l'Instruction, furent répartis dans les différentes académies afin d'être intégrer dans les internats des collègues et des lycées. «Jusqu'au 14 février 1916, 1148 élèves étaient installés dans 43 écoles » tandis que le rapport « du 26 juin 1916 » montre « déjà 3 300 élèves » (TRGOVČEVIĆ 2010 : 366) accueillis dans les établissements français. D'après Slobodan Vitanović, il y aura près de 4000 écoliers scolarisés en France à la fin de la Grande Guerre (VITANOVIĆ 2019).

La ville de Poitiers, ses collèges et lycées ainsi que l'Université de Poitiers vont offrir leur hospitalité à « deux groupes d'enfants serbes » et « le recteur de l'Académie » va encourager « leurs camarades français à bien les accueillir :

militairement dès les premières années de la Grande Guerre. Par conséquent durant les années d'occupation de la Serbie (1916-1918) et la fuite de ses citoyens, le plus grand nombre de ces derniers cherchait refuge en France. Au sein de cette population provisoire il y avait des soldats en grand nombre, blessés, épuisés ou malades. Le second groupe fut celui des élèves et des étudiants, autorisés à poursuivre leurs études en France par la résolution de l'Assemblée Nationale française du 26 novembre 1915. Le troisième groupe consistait en réfugiés civils, particuliers ou avec famille, dont des hommes politiques, qui ont volontairement ou en raison de leurs missions, surtout militaires, suivi l'armée serbe et trouvé refuge sur le sol français. (TRGOVČEVIĆ 2010 : 362). 
'Je vous demande, dit-il, de leur venir en aide. Supposez que les Boches eussent parvenues chez nous ; que vous eussiez, vous aussi, été obligés, abandonnant tout, de fuir devant leurs hordes sauvages et que vous n'eussiez enfin trouvé d'abri qu'à l'étranger ; n'auriez-vous pas été heureux qu'on vous accueillît comme des frères ?’» (AUGUSTIN, SIMMAT 2016 : 11).

\section{Les archives de l'Association Générale des Étudiants de Poitiers (A.G.E.)}

Avant de présenter les résultats des recherches effectuées dans les archives de l'Association générale des étudiants de Poitiers et dans les Archives départementales, il serait propice de mentionner quelques événements historiques précèdent l'arrivée des jeunes Serbes, et notamment la déclaration de Corfou signée le 9 novembre 1916 entre la France et la Serbie. A la suite de cette convention des groupes d'étudiants et d'intellectuels serbes viennent de manière organisée en France qui, pour sa part, soutient vivement la création d'un État commun pour tous les peuples des Slaves du Sud. C'est d'ailleurs une des raisons pour laquelle l'alliée serbe encourage l'arrivée des étudiants, persuadé que leur scolarisation en France permettra de former une élite intellectuelle et politique pour le futur État.

Une mobilisation générale s'organise en France pour aider la Serbie qui combattait alors contre les Autrichiens et les Bulgares sur le Front Sud. C'est aussi dans cet esprit que les intellectuels français de l'époque comme Victor Bérard 4 , le fondateur du " Comité franco-serbe », ou Albert Sarraut donnent plusieurs conférences pour apporter les informations sur la Serbie afin de sensibiliser l'opinion public sur le sort tragique du peuple serbe. C'est ainsi que fut organisé une Journée serbe 5 le 25 juin et le 2 juillet 1916 dans le pays entier dont le but était de récupérer les fonds nécessaires pour accueillir les jeunes refugiés.

Le département de la Vienne a également répondu à l'appel national et a récolté des dons destinés aux élèves serbes. Selon les résultats extraits des Archives Départementales (ARCHIVES DV : R7 34), parmi les journées de bienfaisance organisées dans la région poitevine, il y eut aussi celle consacrée aux Serbes où la somme collectée fut d'environ 15000 francs.

En novembre 1916 le département de la Vienne reçoit également un important contingent de Serbes. A part neuf cultivateurs travaillant aux

\footnotetext{
${ }^{4}$ Spécialiste de philologie classique et traducteur des épopées d'Homère, Victor Bérard est aussi l'auteur de plusieurs études concernant les peuples balkaniques et notamment les Serbes. En 1915 il publie dans les Annales de Paris (janvier 1915 et octobre 1915) deux textes, le premier intitulé Pour la Serbie et le deuxième Dans les Balkans : La Serbie.

${ }^{5}$ Voir les posters de l'époque sur le site LES POSTERS 1914-1918.
} 
Ormes et un ouvrier employé à la manufacture d'armes de Châtellerault, la plupart de ces réfugiés sont des étudiants ou des intellectuels. Ils s'inscrivent à l'Université de Poitiers et deviennent membres de l'Association générale des étudiants de Poitiers. Selon certaines sources, particulièrement celle provenant du commissaire spécial Richelmi (ARCHIVES DV : R7 18, le 22 août 1918), la présence des étudiants serbes dans le département de la Vienne suscita l'inquiétude des autorités locales. Celles-ci craignaient que ces jeunes serbes ne soient à l'origine de conflits politiques car ils n'étaient pas tous monarchistes ; parmi eux, il y avait aussi ceux qui « rêvaient pour la Serbie d'un régime républicain d'une nuance rappelant celle de notre parti radicalsocialiste » (ARCHIVES DV : R7 18, le 22 août 1918). Dans l'ensemble de la communauté étrangère de l'époque " seuls les Serbes paraissent jouer un rôle, politique celui-là, concernant d'ailleurs plus leur nation que la Vienne elle-même » (VINATIER 1984 : 101).

Les archives de l'Association générale des étudiants de Poitiers, fondée le 7 juin 1889, sont déposées depuis quelques années à la Bibliothèque de l'Université de Poitiers ${ }^{6}$. Il s'agit donc de 18 boîtes contenant les documents qui donnent aussi bien des renseignements sur les membres de l'Association que sur leurs activités depuis sa fondation jusqu'aux années 1970.

Concernant l'objet de notre étude, la période entre 1916 et 1918, nous avons examiné les documents déposés dans la boîte titrée «A. G. E. 4 ». Dans celle-ci nous avons trouvé quatre livres contenant les archives entre 1889 et 1945. Le premier livre couvre la période de 1889-1919, le second celle de 1919-1930, le troisième de 1930-1939 et le dernier livre date de 1939-1945.

$\mathrm{Au}$ commencement de leurs activités, les différents comités de l'Association ne fournissent pas dans leurs notes beaucoup d'informations sur l'organisation ou des activités; des renseignements se trouvant dans les cahiers de l'Association portent surtout sur les membres de l'organisation en précisant les noms des présidents, des vice-présidents, des secrétaires ou des délégués (ARCHIVES A.G.E. : boîte 4). La liste des étudiants adhérents apparaît plus tard et varie selon l'année scolaire et les nombres d'inscriptions ; pour illustrer l'ampleur de cette association, citons quelques données précises :

\begin{tabular}{|l|l|}
\hline \multicolumn{1}{|c|}{ Année scolaire } & \multicolumn{1}{c|}{ Nombre d'étudiants associés } \\
\hline $1896-1897$ & 123 \\
\hline $1898-1899$ & 154 \\
\hline $1899-1900$ & 112 \\
\hline $1900-1901$ & 224 \\
\hline $1901-1902$ & 235 \\
\hline $1902-1903$ & 116 \\
\hline
\end{tabular}

${ }^{6}$ Selon les responsables de cette bibliothèque, nous sommes les premiers à les consulter. 
Philologia Mediana

\begin{tabular}{|l|l|}
\hline $1903-1904$ & 121 \\
\hline $1904-1905$ & 122 \\
\hline $1905-1906$ & 145 \\
\hline $1907-1908$ & 69 \\
\hline $1908-1909$ & 94 \\
\hline $1909-1910$ & 119 \\
\hline $1910-1911$ & 98 \\
\hline $1911-1912$ & 122 \\
\hline $1912-1913$ & 190 \\
\hline $1913-1914$ & 150 \\
\hline $1914-1915$ & 45 \\
\hline $1915-1916$ & 49 \\
\hline $1916-1917$ & 80 \\
\hline
\end{tabular}

Les membres de l'Association sont regroupés et notés selon leur numéro de carte d'inscription. Les informations portent aussi sur la date et le lieu de naissance, l'adresse actuelle, la faculté et le numéro d'inscription de chaque étudiant. Les deux dernières rubriques sont réservées à la signature de l'adhérant et aux renseignements divers. En ce qui concerne le montant de la cotisation, il n'est pas possible de savoir ni la somme exacte ni son évolution. L'indice payé figurant souvent dans la rubrique des renseignements divers ainsi que le poste de trésorier qui était régulièrement accordé à l'un des membres du Comité d'organisation informent uniquement sur l'existence de celle-ci. Le seul document qui porte sur le montant de la cotisation annuelle est celui de la réunion générale du 3 avril 1906 qui dit que « la cotisation sera 12 francs de plus payable à l'inscription » (ARCHIVES A.G.E. : boîte 4).

Les archives de l'Association Générale des Étudiants tracent également l'arrivée des étudiants serbes à l'Université de Poitiers. En effet, une semaine après la signature de la convention de Corfou, un groupe d'étudiants serbes débarque à Poitiers. Le commissaire spécial Richelmi écrit à propos de leur arrivée : "A la suite de la convention signée le neuf novembre 1916 à Corfou entre la France et la Serbie, des groupes d'étudiants serbes furent envoyés dans nos centres universitaires vers le quinze novembre suivant »(ARCHIVES DV : R7 18, le 22 août 1918). Selon les mêmes sources " l'université de Poitiers fut appelée ainsi à recevoir un groupe qui n'a cessé depuis de compter une moyenne de cinquante-cinq étudiants, inscrits aux facultés de droit, de science et de lettres de cette ville » (ARCHIVES DV : R7 18, le 22 août 1918). En consultant les archives de l'Association Générale des Étudiants de Poitiers nous avons pu constater que parmi 80 étudiants inscrits dans l'Association pour l'année scolaire 1916-1917, il y avait 45 étudiants serbes (ARCHIVES A.G.E. : boîte 4). Leur enregistrement dans le fichier de 
l'A.G.E. commence par le numéro 33 et date du 16 novembre 1916. Selon le registre de l'Association, ils étaient tous inscrits à la Faculté de Droit sauf deux étudiants inscrits à la fois en lettres et en droit.

En comparant les données des archives départementales avec celles de l'Association on peut constater une divergence dans le nombre d'étudiants arrivés et dans le choix de leurs études. Selon le commissaire spécial Richelmi, il y avait 55 étudiants tandis que le registre de l'Association en donne 45. Selon lui, ces jeunes serbes ont été inscrits dans les facultés de droit, de science et de lettres et pourtant les archives de l'Association confirment que presque tous étaient inscrits en droit. Le manque de concordance s'explique peut-être par le fait que tous les étudiants inscrits à l'Université de Poitiers ne devinaient pas automatiquement les membres de l'Association. Mais cela n'est qu'une hypothèse parce que les archives de l'Association ne donnent aucun document qui pourrait expliquer le règlement d'inscription. De l'autre côté, il est possible d'envisager que le commissaire spécial Richelmi en comptant 55 étudiants serbes dans la région comptait également ces neuf cultivateurs travaillant aux Ormes et l'ouvrier employé à Châtellerault.

Dans l'année scolaire 1917-1918, le nombre d'étudiants serbes à Poitiers gravite autour de même chiffre que l'année précédente. Le registre montre que parmi 45 membres de l'Association, il y avait 39 adhérents serbes (ARCHIVES A.G.E. : boîte 4). Cette fois aussi, les élèves serbes sont tous inscrits à la Faculté de droit et logés au centre ville : Rue de la Cathédrale, Grand' Rue, Chaudron d'or, Place de la Liberté, rue Gambetta, rue Magenta etc. La plupart entre eux furent déjà inscrit à l'Université de Poitiers en 1916. Parmi les noms qui figurent sur les listes des membres de l'Association, on peut distinguer celui de Théophilo Djurovitch, étudiant en droit, enregistré sous le numéro 54 en 1916 et sous le numéro 26 en 1917. Il s'agit en effet d'un activiste politique et, selon les archives départementales, c'est à son initiative qu'un mouvement politique d'allure révolutionnaire se forma parmi les étudiants serbes. Téophilo Djurovitch luttait pour le respect des aspirations légitimes de tous les peuples des Slaves du Sud. Il était aussi très favorable à la création d'un État yougoslave.

Les registres de l'Association montrent que la présence des étudiants serbes à l'Université de Poitiers ne se maintiendra pas trop long temps ; en effet, les noms des étudiants serbes apparaissent dans les listes uniquement entre 1916 et 1918. Déjà en 1918-1919, parmi 87 membres de l'Association, il n'y avait aucun étudiant serbe (ARCHIVES A.G.E. : boîte 4). Le tableau suivant illustre notamment cette tendance : 
Philologia Mediana

\begin{tabular}{|l|l|l|}
\hline \multicolumn{1}{|c|}{ Année scolaire } & \multicolumn{1}{|c|}{$\begin{array}{c}\text { Nombre d'étudiants } \\
\text { associés }\end{array}$} & Nombre d'étudiants serbes \\
\hline $1916-1917$ & 80 & 45 \\
\hline $1917-1918$ & 45 & 39 \\
\hline $1918-1919$ & 87 & 0 \\
\hline $1919-1920$ & 98 & 1 \\
\hline $1920-1921$ & 89 & 0 \\
\hline $1921-1922$ & 252 & 4 \\
\hline $1922-1923$ & 150 & 0 \\
\hline $1923-1924$ & 200 & 0 \\
\hline $1924-1925$ & 194 & 0 \\
\hline $1925-1926$ & 240 & 0 \\
\hline $1926-1927$ & 193 & 0 \\
\hline $1927-1928$ & 155 & 2 \\
\hline
\end{tabular}

Après deux ans d'étude à l'Université de Poitiers la plupart des étudiants serbes rentrèrent chez eux sauf quelques-uns qui restèrent dans la région. C'est le cas de Tochitch Georges, étudiant en droit, inscrit au registre de l'Association sous le numéro 75 en 1916-1917 et sous le numéro 18 en 19171918. Son descendant direct Tochitch Stanislas, étudiant en Faculté des lettres et des langues, s'inscrit pour l'année scolaire 1999-2000 à l'Université de Poitiers avec un désir bien ferme de découvrir la langue de son arrière grandpère : Stanislas s'inscrit en serbo-croate comme langue d'option.

\section{Conclusion}

Le passage bref des étudiants serbes dans la région de la Vienne est marqué par le contexte général des événements historiques de l'époque. Cependant, il illustre une tendance avérée depuis le XIX ${ }^{\mathrm{e}}$ et se confirmant tout au long du $\mathrm{XX}^{\mathrm{e}}$, une affinité et une attirance particulière des élites intellectuelles serbes pour la culture et la civilisation françaises. L'influence française, comme le constate Vitanović (2019), "véhiculée par le savoir, les métiers, les idées, les conceptions, les méthodes, ou encore la lecture d'ouvrages spécialisés et l'adoption de critères français ", s'intensifie davantage avec le temps et les générations suivant la Grande Guerre afin de devenir un modèle culturel à reproduire. Paris, qui fut le centre culturel de l'émigration serbe pendant la Première guerre mondiale, restera le repère constant autour duquel graviteront des intellectuels et des artistes serbes et yougoslaves.

L'analyse des archives de l'Association Générale des Étudiants de Poitiers, montre certain ralentissement dans la coopération universitaire afin de devenir inexistante. C'est une des raisons pour laquelle l'Union nationale 
des étudiants de France (U.N.E.F.) tenta après la Deuxième Guerre mondiale d'instaurer les rapports avec les universités des pays de l'Europe de l'Est. C'était aussi le motif du voyage de leurs représentants à Belgrade et à Zagreb en 1951. Dans les archives de l'Association de Poitiers, il y a également les comptes rendus de ces deux séjours effectués par la délégation française qui montrent la volonté et le désir des deux pays de renouveler les relations entre leurs universités.

Le tout dernier renouveau dans le domaine de la coopération universitaire franco-serbe date de 2016. En effet, dans la cadre du programme européen Erasmus Plus KA107, les échanges des étudiants et des enseignants ont été mis en place permettant ainsi de marquer de façon remarquable l'anniversaire centenaire depuis l'arrivée des premiers étudiants serbes en France mais aussi à Poitiers. C'est ainsi que l'Université de Poitiers, porteur de ce nouveau projet de coopération, accueille de nouveau dans ses locaux les étudiants des universités de Niš, de Belgrade, de Novi Sad, mais également ceux venant des universités de Sarajevo, de Sarajevo Est, de Banja Luka, etc. L'évolution de la collaboration universitaire franco-serbe se confirme davantage avec le tout dernier projet de double diplomation dans le domaine du master FLE conçu en 2018 et réalisé entre l'Université de Poitiers et l'Université de Niš.

Le programme européen Erasmus Plus KA107, mis en place un siècle plus tard, contribue-t-il, de façon symbolique, à marquer le centenaire de l'alliance culturel franco-serbe en faisant venir des dizaines d'étudiants serbes au département de la Vienne et à l'Université de Poitiers ? Les nouvelles générations franco-serbes nous diront...

\section{Bibliographie}

ARCHIVES A.G.E. : Archives de l'Association générale des étudiants de Poitiers. Poitiers : Bibliothèque universitaire de l'Université de Poitiers.

ARCHIVES DV : Archives départementales de la Vienne. R7 18 et R7 34.

ARNAUTOVIĆ 1919 : ARNAUTOVIĆ, Aleksandar. Les phares. Paris : Ligue des universitaires serbo-croato-slovènes, 1919.

AUGUSTIN, SIMMAT 2016 : AUGUSTIN, Jean-Marie et Gérard SIMMAT. « L'accueil d'enfants réfugiés serbes ». Centre Presse 21. 01. 2016 : 11.

BATAKOVIĆ 2006 : BATAKOVIĆ, Dušan. « Les Serbes dans la Première Guerre mondiale : 1916-1918». Histoire du peuple serbe. Dir. : Dušan Bataković. Traduit du serbe par Ljubomir Mihailović. Lausanne : L’Âge d'Homme, 2006, 245-258. <https://serbica.u-bordeaux-montaigne.fr/ index.php/158-archives/archives/787-batakovic-dusan-t-les-serbesdans-la-premiere-guerre-mondiale-ii-1916-1918>. 19. 09. 2019. 
CHAUVIN 2018 : CHAUVIN, Jacques. « À Poitiers on croit à la future Yougoslavie ». La nouvelle république 30. 03. 2018. <https://www. lanouvellerepublique.fr/poitiers/a-poitiers-on-croit-a-la-futureyougoslavie>. 17. 09. 2019.

DUCASSE 1920 : DUCASSE, André. Balkans 14-18 ou Le chaudron du diable. Paris : Laffont, 1920.

GASCOIN 1919 : GASCOIN, Eugène. Les victoires serbes de 1916. Paris : Bossard, 1919.

IBROVAC 2014 : IBROVAC, Miodrag. « Les élèves serbes en France ». Serbica $\mathrm{n}^{\circ}$ 8-9 (juillet 2014).<https://serbica.u-bordeauxmontaigne.fr/ index.php/archives/158 archives/archives/783-ibrovac-miodrag-leseleves-serbes-en-france-1930>. 02. 06. 2019.

LES POSTERS 1914-1918 : «1914-1918 : Reims dans la Grande Guerre ». $<$ https://reims 1418.wordpress.com/category/journal-de-la-grandeguerre/page/133/>. 19. 09. 2019.

MOROKVAŠIĆ-MÜLLER 1998 : MOROKVAŠIĆ-MÜLLER, M. « Il était une fois une communauté des Yougoslaves en France ». Histoire de l'immigration en France au XXe siècle. Dir. : L. Gervereau, P. Milza et E. Temime. Paris : Bibliothèque de documentation international contemporaine, Musée d'Histoire Contemporaine - Somogy : Ed. d'Art, 1998.

MOULINS 1917 : MOULINS, Amédée. Université française et la jeunesse serbe. Bruxelles-Paris : G. Van Oest, 1917. <https://archive.org/details/ luniversitfran00moul/page/122>. 17. 09. 2019.

NEDELKOVITCH 1960 : NEDELKOVITCH, Dushan. « Les principaux savants Yougoslaves au $\mathrm{XIX}^{\mathrm{e}}$ siècle ». Revue d'histoire des sciences (1960) : 317-323. <https://www.persee.fr/doc/rhs_0048-7996_1960_ num_13_4_3887>.10.09.2019.

PAVLOVIĆ 1988 : PAVLOVIĆ, Mihajlo. Témoignages français sur les Serbes et la Serbie 1912-1918. Belgrade : Narodna Knjiga, 1988.

RFSS 1980 : Les Rapports entre la France et les Slaves du Sud. Premier Colloque historique franco-yougoslave, Paris (24-27 avril 1980). Institut national des langues et civilisations orientales. Centre d'études des civilisations de l'Europe centrale et du sud-est. Paris : Langues'O, 1980.

SRETENOVIĆ 2018 : SRETENOVIĆ, Stanislav. « Histoire et mémoire des relations franco-serbes : un héritage de la Première Guerre mondiale ». H 14-18 Mission centenaire 25. 04. 2018. < http://centenaire.org/fr/ espace-scientifique/histoire-et-memoire-des-relations-franco-serbes-unheritage-de-la-grande-guerre>. 10. 09. 2019.

TRGOVČEVIĆ-MITROVIĆ 1990 : TRGOVČEVIĆ-MITROVIĆ, Ljubinka. « Paris comme centre culturel de l'émigration serbe pendant la Première guerre mondiale ». Зборник радова са научног скупа 
„Југословенско-франиуски односи: поводом 150 година од отварања првог франиуског конзулата у Србији”. Одговорни уредник: Славко Терзић. Београд: Историјски институт, 1990, 198-214.

TRGOVČEVIĆ 2010 : TRGOVČEVIĆ, Ljubinka. « Les Serbes en France durant la Première Guerre mondiale ». La Serbie et la France. Une alliance atypique : les relations politiques, économiques et culturelles 1870-1940. Dir. : D. T. Bataković. Belgrade : Institut des Etudes balkaniques, ASSA, 2010, 361-378. <https://serbica.u-bordeauxmontaigne.fr/index.php/archives/158-archives/archives/742-trgovcevicljubinka-les-serbes-en-france-durant-la-premiere-guerre-mondiale >. 19. 09. 2019.

TROUDE 2010 : TROUDE, Alexis. " La France et la Serbie 19151918 : coopération militaire, implantation économique et échanges culturels ». La Serbie et la France. Une alliance atypique : les relations politiques, économiques et culturelles 1870-1940. Dir. : D. T. Bataković. Belgrade : Institut des Etudes balkaniques, ASSA, 2010, 337-360. $<$ https://serbica.u-bordeaux-montaigne.fr/index.php/archives/158archives/archives/743-troude-alexis-la-france-et-la-serbie-1915-1918cooperation-militaire-implantation-economique-et-echanges-culturels $>$. 19. 09. 2019.

VINATIER 1984 : VINATIER, F. Le Département de la Vienne pendant la Première guerre mondiale : août 1914 - novembre 1918. Mémoire de maîtrise. Poitiers : Université de Poitiers, 1984.

VITANOVIĆ 2019 : VITANOVIĆ, Slobodan. "Cent cinquante ans d'influence française sur la culture serbe ». Serbica n ${ }^{\circ} 25$ (mai 2019). $<$ https://serbica.u-bordeaux-montaigne.fr/index.php/revues/1250slobodan-vitanovic-cent-cinquante-ans-d-influence-francaise-sur-laculture-serbe>. 07. 09. 2019.

Сања Б. Бошковић-Данојлић

\section{СРПСКИ СТУДЕНТИ НА УНИВЕРЗИТЕТУ У ПОАТЈЕУ ЗА ВРЕМЕ ПРВОГ СВЕТСКОГ РАТА}

Што се тиче Француске, земље која је указала гостопримство српским, а затим и југословенским емигрантима, може се рећи да је њихов број варирао у различитим периодима. Интезивнији миграциони таласи, укључујући посебно младо становништво - ученике и студенте, забележени су за време Првог светског рата. Након повлачења војске и протеривања српског народа који је прешао Албанију у зиму 1915/1916, број српских ученика и студената који ће пристићи у Француску нарочито се повећао 
1916. године. Град Поатје, његови колеџи и средње школе, као и Универзитет у Поатјеу, понудиће гостопримство младим српским избеглицама. Архив Генералног удружења студената са Универзитета у Поатјеу, које је основано 7. јуна 1889. године, показује не само померања у броју придошлих српских школараца током Првог светског рата, већ сведочи и о специфичном историјском тренутку у француско-српским политичким и културним односима.

Кључне речи: Први светски рат, српске избеглице, српски студенти, Поатје 